(15), (16) between operations in the $I$-ring, and in $I F$, and the correspondence between $I F$ and a ring of an abstract field, the theorem is proved.

The element $X(x)=\sum \xi(n) x^{n}$ of the $I$-ring is now defined to be regular or irregular according as $X^{\prime}(x)=\sum \xi^{\prime}(n) x^{n}$, where $\xi \xi^{\prime}=\eta$, is or is not in the $I$-ring.

Let $A(x)=\sum \alpha(n) x^{n}$ be any element of the $I$-ring, and $B(x)=\sum \beta(n) x^{n}$ any regular element of the $I$-ring. Write $B^{\prime}(x)=\sum \beta^{\prime}(n) x^{n}$, where $\beta \beta^{\prime}=\eta$. Then the I-quotient $A(x) / B(x)$ of $A(x)$ by $B(x)$ is defined by

$$
A(x) / B(x)=A(x) B^{\prime}(x) ;
$$

$B^{\prime}(x)$ is called the I-reciprocal of $B(x)$, and we write $B^{\prime}(x)$ $=U(x) / B(x)$. Combining (18), (19), we have the following theorem.

(20) ThEOREM. The set of all elements of the I-ring is an irregular field, say the I-field, in which division is as in (19) and the remaining fundamental operations as in (18); the irregular elements of the I-field are those of the I-ring.

California Institute of Technology

\title{
ON TRI-RHAMPHOIDAL AND BI-OSCNODAL QUINTIC CURVES
}

BY HAROLD HILTON

In a recent paper,* T. R. Hollcroft says "For example, a quintic may have three rhamphoid cusps or two tacnodecusps."

Now it is true that there is just one projectively distinct quintic with three rhamphoid cusps (or two, if we confine ourselves to real projections), namely

$$
\begin{gathered}
x: y: z=t^{2}\left(t-\frac{3}{2}-\frac{1}{2} \sqrt{ } 5\right): t^{2}(t-1)^{2}\left(t-\frac{1}{2}+\frac{1}{2} \sqrt{ } 5\right) \\
:(t-1)^{2}\left(t+\frac{1}{2}-\frac{1}{2} \sqrt{ } 5\right) .
\end{gathered}
$$

* This Bulletin, vol. 35 (1929), p. 847. 
It is the quadratic transform of a three-cusped quartic with respect to a triangle, whose vertices lie on the quartic and each of whose sides passes through a cusp.*

But it is not true that a quintic can have two tacnode-cusps. For, taking $A B C$ as triangle of reference and using suitable homogeneous coordinates, write down the general equation of a quintic with double points at $A$ and $B$ such that the tangents to both branches at these points are respectively $A C$ and $B C$, while these tangents meet the curve four times at $A$ and $B$. Then analyze the equation of the curve to obtain the conditions for the existence of two latent double points at each of $A$ and $B . \dagger$ We see then that there are two types of quintic curve with two real oscnodes $A, B$, and tangents $A C, B C$.

One of them is

or

$$
x\left(x y+a z^{2}\right)^{2}=y\left(x y+b z^{2}\right)^{2},
$$

$$
x: y: z=t\left(t^{2}+b\right)^{2}: t\left(t^{2}+a\right)^{2}:\left(t^{2}+a\right)\left(t^{2}+b\right) .
$$

The analysis shows that for no value of $a$ or $b$ can one of the oscnodes become a tacnode-cusp.

The tangents at the oscnodes $A, B$ meet at an inflexion $C$ of the curve, the tangent being $a^{2} x=b^{2} y$. The remaining tangent from $C$ to the curve, namely $x=y$, has its point of contact on $A B$.

The other type of quintic with oscnodes $A, B$ and tangents $A C, B C$ is

$$
x\left(u+a z^{2}\right)^{2}+y\left(u+b z^{2}\right)^{2}=2 z\left(u+a z^{2}\right)(u+b z)^{2},
$$

where $u \equiv x y-z^{2}$; or

$$
x: y: z=(1-t)\left(b-t^{2}\right)^{2}:(1+t)\left(a-t^{2}\right)^{2}:\left(a-t^{2}\right)\left(b-t^{2}\right) .
$$

The analysis shows that the oscnode at $A$ becomes a tacnodecusp if $a=0$, and so for $B$. But we cannot have two tacnode-cusps, for the curve degenerates if both $a$ and $b$ are zero.

Bedford College,

REgents PARK, LoNdon

* See Hilton's Plane Algebraic Curves, Clarendon Press, p. 120.

† Loc. cit., p. 134. 